\title{
Check! Explore Barriers and Solutions to Data Sharing on BioSense 2.0
}

\author{
Marcus Rennick ${ }^{\star 1}$, David J. Swenson ${ }^{2}$, Stacey Hoferka ${ }^{3}$, Charlie Ishikawa ${ }^{4}$ and Rebecca \\ Zwickl ${ }^{4}$
}

${ }^{1}$ Marion County Public Health Department, Indianapolis, IN, USA; ${ }^{2}$ New Hampshire Department of Health and Human Services, Concord, NH, USA; ${ }^{3}$ Illinois Department of Public Health, Chicago, IL, USA; ${ }^{4}$ International Society for Disease Surveillance, Boston, MA, USA

\section{Objective}

Build upon the findings of a Regional Data Sharing workshop with the larger surveillance community to more clearly describe the benefits, barriers, and needs for data sharing on the BioSense 2.0 platform.

\section{Introduction}

Inter-jurisdictional data sharing can enhance disease surveillance capabilities for local, state, regional and national public health situational awareness and response. BioSense 2.0, a cloud-based computing platform for syndromic surveillance, provides participating local, state and federal health jurisdictions with the ability to share aggregated data; a functionality that is easily activated by selecting an administrative checkbox within the BioSense application. Checking the data-sharing box, however, is a considerable decision that comes with benefits and consequences.

On May 20-21, 2013, nine city, county, and state public health department jurisdictions (mainly from the mid-western region of the U.S.) met to explore data sharing for Heat Related Illness (HRI) surveillance using BioSense 2.0. During the workshop, all participants agreed to share data (using the BioSense 2.0 front-end application) in real-time to investigate HRI trends in regional populations during May-August 2012, evaluated HRI case-definitions, and documented benefits and barriers to inter-jurisdictional data sharing. The workshop was convened by ISDS, in collaboration with the Association of State and Territorial Health Officials (ASTHO), with the support of the U.S. Centers for Disease Control and Prevention. Staff from BioSense programmatic and technical teams were also present for the workshop.

\section{Description}

This roundtable will build upon the workshop findings to develop recommendations for the BioSense Governance Group. Participants will have the opportunity to modify or add to the workshop's list of perceived barriers to inter-jurisdictional data sharing, develop solutions, and contribute data sharing success stories.

Benefits to data sharing identified at the workshop include:

- Cross-border case-finding

-Identifying patterns or trends (local, state, regional, federal)

-Emergency preparedness planning and partner notification

-Estimating an end to an event, based on declining trends in neighboring areas

-Mutual aid

-Ensuring national situational awareness for federal partners

-Hypothesis generation and testing

- Retrospective analysis to improve public health practice

Barriers to data sharing identified at the workshop include:

-Legal considerations regarding protected health information

-Ambiguity as to whom data is shared with and limited granularity in data sharing controls

-Quality of shared data is unknown introducing biases

\section{Audience Engagement}

Is there agreement/disagreement regarding our data sharing benefits? Other benefits to add?

-What barriers do you currently have with sharing data? Solutions to overcome barriers?

-What metadata should be available (and how should it be displayed) to overcome data quality barriers?

-Besides BioSense, does your jurisdiction have other syndromic surveillance systems in place? Is BioSense your primary analytic tool?

-What data quality measures would be integral to proper interpretation of the data?

-Is there agreement or disagreement regarding our recommendations? Other recommendations?

-What user roles, if any, should be identified for data sharing with other jurisdictions?

\section{Conclusions}

Data sharing can benefit a range of public health activities including cross-border outbreak detection, identifying regional and federal health trends, and hypothesis generation and testing

- Convening an in-person Workshop facilitated trust and interpersonal relationship building, as well as the initiation of plans for 13 new data sharing permissions among participants (which included shifts from sharing aggregate to line-level data and increased inter-jurisdictional sharing between $\mathrm{CDC}$ and local health departments versus between state and local prior to the Workshop)

-Data quality and metadata are key; all data users need to understand the context and quality of the data

-Substantial increases in epidemiological knowledge, skills, and abilities were noted from pre and post workshop assessments

-Legal and privacy concerns can block effective data sharing

\section{Keywords}

BioSense; Data Sharing; Collaboration

\section{Acknowledgments}

Workshop participants would like to acknowledge the ISDS staff for facilitating the meeting, sponsored by ASTHO and the CDC, with valuable technical feedback from CDC and RTI staff.

\section{*Marcus Rennick}

E-mail: mrennick@hhcorp.org 